\title{
Infecção hospitalar: controle e disseminação nas mãos dos profissionais de saúde de uma Unidade de Terapia Intensiva
}

\author{
Hospital infection: control and dissemination in the hands of professionals of an Intensive Care Unit \\ Infección hospitalaria: control y diseminación en manos de los profesionales de la salud de una \\ Unidad de Terapia Intensiva
}

\begin{abstract}
Wagner Bechorner Almeida ${ }^{1}$, Neiva Claudete Brondani Machado ${ }^{2 *}$, Andressa Peripolli Rodrigues ${ }^{2}$, Izabel Almeida Alves ${ }^{3}$, Rosane Teresinha Fontana ${ }^{3}$, Rita Fernanda Fernandes Monteiro ${ }^{2}$, Narciso Vieira Soares ${ }^{3}$
\end{abstract}

\section{RESUMO}

Objetivo: Identificar os microrganismos presentes nas mãos dos profissionais de saúde de uma unidade de terapia intensiva e verificar as causas da baixa adesão à higienização das mãos. Método: Estudo qualiquantitativo, exploratório, do tipo transversal. A coleta de dados qualitativos foi por meio de entrevista semiestruturada e os quantitativos por meio da análise de cultura coletada das mãos dos profissionais da saúde da unidade de terapia intensiva. Participaram do estudo enfermeiros, técnicos em enfermagem e médicos. Utilizou-se a análise de conteúdo para os dados qualitativos e os quantitativos por meio da observação do crescimento de microrganismos nas placas de cultura e posterior identificação destes, sendo disponibilizados no Microsoft Office Word 2007, para análise estatística. Resultados: O estudo evidenciou um percentual de contaminação de $33,3 \%$ na categoria profissional de enfermeiro, $46,7 \%$ de técnicos em enfermagem e $100 \%$ no profissional médico. No entanto, a maioria dos participantes afirmam que o ambiente de trabalho proporciona a adesão à higienização das mãos, paradoxalmente observa-se que $50 \%$ da amostra total obtiveram contaminação. A justificativa para a não adesão a lavagem de mãos foi à sobrecarga de trabalho. Conclusão: Observou-se um índice elevado de contaminação, bem como um perfil profissional de resistência para a não adesão à higienização das mãos.

Palavras-chave: Unidades de Terapia Intensiva, Infecção hospitalar, Pessoal de saúde.

\section{ABSTRACT}

Objective: To identify the microorganisms present in the hands of the health professionals of an intensive care unit and to verify the causes of low adherence to hand hygiene. Method: Qualitative, exploratory, crosssectional study. The collection of qualitative data was through a semi-structured interview and the quantitative data were collected through the culture analysis collected from the hands of the health professionals of the intensive care unit. Nurses, nursing technicians and physicians participated in the study. The analysis of the content for the qualitative and quantitative data was done by means of the observation of the growth of microorganisms in the culture plates and later identification of these, being made available in Microsoft Office Word 2007, for statistical analysis. Results: The study showed a percentage of contamination of $33.3 \%$ in the professional category of nurse, $46.7 \%$ in nursing technicians and $100 \%$ in the medical professional. However, the majority of the participants affirm that the work environment provides adherence to hand hygiene, paradoxically it is observed that $50 \%$ of the total sample obtained contamination. The justification for nonadherence to handwashing was to work overload. Conclusion: A high contamination index was observed, as well as a professional resistance profile for non-adherence to hand hygiene.

Keywords: Intensive Care Units, Cross infection, Health personnel.

1 Serviço Nacional de Aprendizagem Comercial (SENAC) e Sistema de Ensino Gaúcho, Santo Ângelo - RS.

2 Instituto Federal de Educação, Ciência e Tecnologia Farroupilha (IFFar), Campus de Santo Ângelo - RS.

*E-mail: neiva.machado@iffarroupilha.edu.br

3 Universidade Regional Integrada do Alto Uruguai e das Missões (URI), Campus de Santo Ângelo - RS. 


\section{RESUMEN}

Objetivo: Identificar los microorganismos presentes en las manos de los profesionales de salud de una unidad de terapia intensiva y verificar las causas de la baja adhesión a la higienización de las manos. Método: Estudio cual-cuantitativo, exploratorio, del tipo transversal. La recolección de datos cualitativos fue a través de una entrevista semiestructurada y los cuantitativos por medio del análisis de cultura recogida de las manos de los profesionales de la salud de la unidad de terapia intensiva. Participaron del estudio enfermeros, técnicos en enfermería y médicos. Se utilizó el análisis de contenido para los datos cualitativos y los cuantitativos por medio fue de la observación del crecimiento de microorganismos en las placas de cultivo y posterior identificación de éstos, estando disponibles en Microsoft Office Word 2007, para análisis estadístico. Resultados: El estudio evidenció un porcentaje de contaminación del 33,3\% en la categoría profesional de enfermero, el $46,7 \%$ en técnicos de enfermería y el $100 \%$ en el profesional médico. Sin embargo, la mayoría de los participantes afirman que el ambiente de trabajo proporciona la adhesión a la higienización de las manos, paradójicamente se observa que $50 \%$ de la muestra total obtuvieron contaminación. La justificación para la no adhesión del lavado de manos fue a la sobrecarga de trabajo. Conclusión: Se observó un alto índice de contaminación, así como un perfil profesional de resistencia para la no adhesión a la higienización de las manos.

Palavras-clave: Unidades de Cuidados Intensivos, Infección hospitalaria, Personal de salud.

\section{INTRODUÇÃO}

O Ministério da Saúde define infecção hospitalar como uma infecção adquirida após a admissão do paciente na unidade hospitalar e que se manifesta durante a internação ou após a alta, esta última quando estiver relacionada com a internação ou procedimentos hospitalares realizados (BRASIL, 1998). Diante disso, para redução dos índices de infecção hospitalar tem-se a higienização das mãos como pilar da prevenção destas infecções (OLIVEIRA et al., 2016).

A microbiota da pele tem duas populações de microrganismos: a residente e a transitória. A residente é de baixa virulência, sendo composta por microrganismos que se multiplicam na pele, ficando estáveis e viáveis por longos períodos, não sendo fácil a sua remoção, mas são inativadas pelo uso de produtos antissépticos. No entanto, devido à descamação natural da pele e a produção de suor, alguns destes microrganismos são movidos para camadas mais superficiais e eliminados no ambiente. Já a transitória é composta por microrganismos que são considerados os principais causadores da maioria das infecções hospitalares, caracterizados pela inabilidade de multiplicar-se na pele, viáveis por curto período e encontrados na superfície da pele, junto a gorduras e sujidades. Por serem removidos facilmente da pele por meio de ação mecânica, estes microrganismos também se espalham facilmente pelo contato (CORDEIRO e LIMA, 2016).

A nível mundial, a infecção hospitalar representa um importante problema de saúde pública, constituindo risco à saúde dos usuários dos hospitais que se submetem aos procedimentos terapêuticos ou de diagnóstico. Sua prevenção e controle dependem, em grande parte, da adesão dos profissionais da área de saúde as medidas preventivas (GIAROLA et al., 2012; BELELA-ANACLETO et al., 2013).

Dentre essas medidas, está a higienização das mãos, que deve ocorrer antes e após o contato com o paciente, mesmo com o uso de luvas, abrangendo toda a superfície das mãos e punhos, utilizando-se de sabão/detergente e/ou antisséptico, dependendo da sujidade. Destaca-se que a higienização correta das mãos contribui para a prevenção e controle de infecções hospitalares (BRASIL, 2009; MOTA et al., 2014; CORDEIRO e LIMA, 2016).

Diante disso, a sensibilização dos profissionais de saúde a respeito da importância da higienização das mãos é imprescindível no contexto hospitalar quando se aborda o controle de infecção, pois a transmissão de infecção pode ocorrer, principalmente, pelas mãos da equipe que assiste ao paciente internado ou por artigos contaminados pelo contato com sangue, secreção ou excretas. A contaminação também pode se dar por meio de gotículas de secreções respiratórias, pelo ar e/ou ainda pelo manuseio incorreto de equipamentos invasivos, como ventiladores mecânicos, cateteres venosos periféricos e centrais, sondas naso e orogástricas ou vesicais (CORDEIRO e LIMA, 2016). 
Portanto, em um ambiente onde há pacientes com quadro clínico debilitado, frágil e suscetível, com alta probabilidade de contrair infecções é consenso que a transmissão por contato desempenha o papel mais importante nesta dinâmica de transmissão. Este perfil caracteriza a internação de pacientes em Unidade de Terapia Intensiva (UTI). Embora as UTIs representem $5 \%$ a $10 \%$ dos leitos de um hospital, o risco de adquirir infecção é estimado entre cinco e dez vezes maior que nas demais unidades (FIGUEIREDO et al., 2013).

O paciente que desenvolver infecção hospitalar, independentemente da fonte ou motivo, elevará seu tempo de permanência internado, o que acarretará maiores custos ao serviço hospitalar e a propensão de desenvolver resistência ao uso de antibióticos para combater microrganismos multirresistentes (CATANEO et al., 2011). Assim, justifica-se este estudo no sentido de suscitar reflexões entre os profissionais da saúde a respeito de manter uma higienização adequada das mãos, no intuito de prevenir infecções hospitalares.

Dessa forma, o objetivo deste estudo foi identificar os microrganismos presentes nas mãos dos profissionais de saúde de uma UTI e verificar as causas da baixa adesão à higienização das mãos.

\section{MÉTODO}

Trata-se de uma pesquisa quali-quantitativa, exploratória, do tipo transversal. Participaram da pesquisa 20 profissionais da área da saúde que atuam na UTI de um hospital referência na região noroeste do Estado do Rio Grande do Sul. O critério de inclusão foi ser profissional da área da saúde da referida unidade e foram excluídos aqueles que estavam afastados do serviço devido a licença ou férias.

A coleta de dados ocorreu em 2013 e para os dados qualitativos utilizou-se a entrevista semiestruturada realizada na própria unidade, em sala reservada para esse fim. O conteúdo das narrativas dos participantes foi gravado por mídia digital a partir da autorização dos sujeitos, transcrito e analisado por meio da análise de conteúdo temática (MINAYO, 2007).

A coleta dos dados quantitativos ocorreu mediante análise dos meios de cultura coletados através de swab estéril das mãos dos enfermeiros, técnicos em enfermagem e médicos atuantes na UTI e, posteriormente, semeados em Ágar cromogênico, onde a susceptibilidade aos microrganismos Pseudomonas aeruginosa, Acinetobacter baumanni, Escherichia coli, Klebsiella pneumoniae e Staphylococcus aureus foi avaliada. A análise foi realizada por meio da observação do crescimento de microrganismos nas placas de cultura por quatro dias e posterior identificação dos microrganismos presentes. Os dados foram disponibilizados em tabelas do Microsoft Office Word 2007 para análise estatística.

O estudo foi submetido ao Comitê de Ética conforme determina a Resolução 466/12 do Conselho Nacional de Saúde recebendo aprovação sob o número do protocolo 261.496. Aos participantes do estudo foi entregue o Termo de Consentimento Livre e Esclarecido em duas vias, permanecendo uma com os participantes e outra com os pesquisadores e para preservar o anonimato dos sujeitos foram utilizados nomes fictícios de microrganismos.

\section{RESULTADOS E DISCUSSÃO}

Com relação a análise de culturas, de um total de 20 participantes, $75 \%(n=15)$ representam a categoria de técnico em enfermagem, $15 \%(n=3)$ enfermeiros e $10 \%(n=2)$ médicos. A tabela 1 evidencia que a categoria profissional enfermeiro obteve uma contaminação $(n=1)$, perfazendo um percentual de contaminação de $33,3 \%$, a categoria de técnicos em enfermagem obteve sete contaminações com $46,7 \%$ e a categoria profissional médico apresentou duas contaminações o que corresponde a $100 \%$ de ocorrência.

A tabela 2 indica os microrganismos presentes na análise e o percentual de contaminação quanto à categoria profissional. A categoria profissional enfermeiro obteve um percentual de contaminação de 33,3\% com presença de Staphylococcus aureus. 
Tabela 1: Prevalência de contaminação quanto à categoria profissional atuante na UTI.

\begin{tabular}{llc}
\hline Categoria Profissional & $\mathbf{N}^{\mathbf{0}}$ & $\mathbf{\%}$ \\
\hline Enfermeiro $(\mathrm{n}=3)$ & 01 & $33,3 \%$ \\
Técnico de Enfermagem $(\mathrm{n}=15)$ & 07 & $46,7 \%$ \\
Médico $(\mathrm{n}=2)$ & 02 & $100 \%$ \\
\hline Total $(\mathbf{n}=\mathbf{2 0})$ & $\mathbf{1 0}$ & $\mathbf{5 0 \%}$
\end{tabular}

Fonte: Dados da pesquisa, 2013.

Na categoria técnico em enfermagem, os achados selecionados indicaram um percentual de contaminação de 13,3\% para Acinetobacter baumani, 20\% para Staphylococcus aureus e 6,7\% para Escherichia coli e Klebsiella pneumoniae. A análise percentual total de contaminação perfez $60 \%$ da amostra $(n=9)$. A categoria médico obteve achados selecionados de $50 \%$ para Staphylococcus aureus e achados casuais de $50 \%$ para staphylococcus coagulase negativa e $100 \%$ para candida albicans.

Segundo o manual de higienização dos serviços de saúde da Agência Nacional de Vigilância Sanitária (ANVISA), a microbiota transitória coloniza a camada mais superficial da pele, sendo representada, tipicamente, pelas bactérias Gram-negativas, como enterobactérias (Ex: Escherichia coli), bactérias não fermentadoras (Ex: Pseudomonas aeruginosa), além de fungos e vírus. Os patógenos hospitalares mais relevantes são: Staphylococcus aureus, Staphylococcus epidermidis, Enterococcus spp., Pseudomonas aeruginosa, Klebsiella spp.. Enterobacter spp. e leveduras do gênero Candida (BRASIL, 2007; CORDEIRO e LIMA, 2016).

Esta relevância é observada na tabela 2 que indica a existência de microrganismos e o percentual de contaminação quanto à categoria profissional. Observa-se que em mais de uma categoria houve crescimento de um ou mais microrganismo no mesmo meio de cultura.

Entender o trabalho realizado em UTI compreende perceber a complexidade deste contexto profissional. O perfil da unidade requer trabalhadores envolvidos com o cuidado de forma muito intensa, pois trata-se de uma unidade com pacientes em estado grave, com patologias de base primárias, secundárias e terciárias em um ambiente de procedimentos invasivos necessários a reabilitação.

Tabela 2: Microrganismos presentes nas mãos dos profissionais atuantes em UTI.

\begin{tabular}{lccc}
\hline \multicolumn{1}{c}{ Microrganismos } & $\begin{array}{c}\text { Enfermeiro } \\
(\mathbf{n}=\mathbf{3})\end{array}$ & $\begin{array}{c}\text { Téc. em enferm. } \\
(\mathbf{n = 1 5})\end{array}$ & $\begin{array}{c}\text { Médico } \\
(\mathbf{n}=\mathbf{2})\end{array}$ \\
\hline $\begin{array}{l}\text { Achados selecionados } \\
\text { Pseudomonas aeruginosas }\end{array}$ & - & - & - \\
$\quad \begin{array}{c}\text { Acinetobacter baumani } \\
\text { Escherichia coli }\end{array}$ & - & $2(13,3 \%)$ & - \\
$\quad$ Klebsiella pneumoniae & - & $1(6,7 \%)$ & - \\
$\quad$ Staphylococcus aureus & - & $1(6,7 \%)$ & $1(50 \%)$ \\
\hline $\begin{array}{l}\text { Achados casuais } \\
\text { Staphylococcus coagulase negativa }\end{array}$ & $1(33,3 \%)$ & $3(20 \%)$ & $1(50 \%)$ \\
Candida albicans & - & $2(13,3 \%)$ & $2(100 \%)$ \\
\hline
\end{tabular}

Fonte: Dados da pesquisa, 2013. 
Algumas reflexões são necessárias, principalmente, quando a maioria dos participantes afirmam que o ambiente de trabalho proporciona a adesão à higienização das mãos, disponibilizando condições para sua realização efetiva, em contrapartida, observa-se que $50 \%$ da amostra total obtiveram contaminação. Este fator isolado condiciona a percepção de que os profissionais reconhecem que a higienização é promovida na instituição, todavia, deixam de realizar este procedimento de forma adequada e em periodicidade recomendada no ambiente de terapia intensiva.

As mãos dos profissionais de saúde representam o principal veículo de transmissão de microrganismos, sendo o principal fator determinante das infecções hospitalares. Por isso, a higienização das mãos não deve ser vista apenas como uma prática opcional, mas como obrigação, fundamental para a garantia da assistência segura (OLIVEIRA e PINTO, 2018).

A UTI é um ambiente com pacientes que apresentam quadro clínico debilitado e suscetível a infecções (STUBE et al., 2013). Quando se alia a alta probabilidade de contrair e ou transmitir infecções, o consenso de que a transmissão por contato existe, a precária desinfecção das mãos e a baixa adesão dos profissionais a higienização, pode ser desencadeada uma dinâmica de transmissão infecciosa (ROCHA e LAGES, 2016).

Fatores intrínsecos ao processo de cuidar envolvem a avaliação de problemas institucionais emergentes e desencadeadores de risco ao paciente hospitalizado. A falta de comprometimento, a sobrecarga de trabalho e o pouco conhecimento da técnica de higienização das mãos desempenha o papel mais importante nesta dinâmica de transmissão (BELELA-ANACLETO et al., 2017).

Além disso, diversos motivos são apontados para a baixa adesão à higienização das mãos pelos profissionais, destacando-se a falta de motivação, ausência de pias próximas ao paciente e de recursos materiais adequados, reações cutâneas nas mãos, falta de tempo e até mesmo a falta de informação sobre a importância das mãos na contaminação cruzada (ALMEIDA et al., 2017; BELELA-ANACLETO et al., 2017; OLIVEIRA e PAULA, 2014).

Apesar de sua importância epidemiológica, promover a adesão para a higienização das mãos é um desafio. A indisponibilidade e a dificuldade de acesso aos insumos necessários para essa prática constituem barreiras relevantes nesse processo, sendo que o preparo da instituição, por meio da provisão de infraestrutura e do fornecimento de recursos constitui requisito fundamental na promoção da higienização das mãos (BELELA-ANACLETO et al., 2013).

Destaca-se também que a realização correta da técnica de higienização das mãos no cotidiano hospitalar implica em uma postura profissional consciente e que traduz benefícios ímpares ao paciente, pois se trata da principal forma de evitar a disseminação de microrganismos e, consequentemente, a infecção cruzada. Assim, a promoção e a realização da higienização das mãos podem reduzir as taxas de infecção nos serviços de saúde, por se tratar de uma das medidas mais importantes para redução da transmissão cruzada de microrganismos e, consequentemente, das taxas de infecção (BRASIL, 2009).

Estudos têm demonstrado a importância da referida prática nessa redução das infecções hospitalares. A maioria dos especialistas em controle de infecções afirma que esse procedimento é o meio mais simples e eficaz de prevenir a transmissão de microrganismos no ambiente assistencial (MOTA et al., 2014).

O contato do profissional com o paciente, com artigos e equipamentos contaminados durante o cuidado prestado deve ser vislumbrado como fator gerador de preocupação. Neste sentido, técnicas criteriosas de higiene e descontaminação são emergentes, e passam a serem visualizadas por quem identifica esta condição como agravante da condição de saúde, conforme apontam as falas dos profissionais:

\section{"[...] prevenir infecções, doenças para si próprio e doenças cruzadas [...]" Pseudomonas aeruginosas}

"[...] vital para a nossa saúde e de nossos familiares e do paciente [...]" Acinetobacter baumani

O bem-estar da família do trabalhador de saúde merece tamanha atenção quanto à família do paciente, uma vez que ambos possuem a possibilidade de contaminação. A diferença crucial desta dicotomia é que 
cessa a preocupação dos familiares do paciente quando este se recupera e tem alta da UTI e/ou do hospital, enquanto a família dos profissionais de saúde está em constante risco (VOLQUIND et al., 2013).

O entendimento dos profissionais da saúde quanto à importância das mãos na cadeia de transmissão das infecções hospitalares existe, todavia, passivamente, não realizam a técnica correta para sua efetivação. Observa-se uma deficiência em descrever todos os passos preconizados e a falta de rigor na sequência das etapas, o que proporciona risco iminente de transmissão de patógenos:

\section{"[...] sempre deixar a água corrente de distal para proximal [...]" Escherichia coli}

A técnica de higienização das mãos é, na maioria das vezes, inadequada pelo esquecimento de algumas etapas desse procedimento devido sobrecarga de serviço, havendo preocupação com a quantidade e não com a qualidade. Existe uma maior incidência de infecções hospitalares, cerca de $20 \%$ a mais, naquelas unidades em que a prática da higienização das mãos é reduzida ou realizada de maneira incorreta (MOTA et al., 2014)

Em se tratando de unidade de cuidados intensivos, são elevadas as exigências, devido ao perfil dos pacientes atendidos e da necessidade da tomada de decisão imediata pelo agravante da condição clínica dos pacientes. Além da complexidade dos cuidados prestados, a exigência de profissionais com visão, atitude e conhecimento apurado gera desgaste físico, psicológico e emocional no trabalhador que se vê em meio a situações graves:

\section{"[...] a pressa, sobrecarga de tarefas, emergências [...]" Klebsiella pneumoniae}

"[...] a falta de tempo, quando acontece intercorrências com algum paciente [...]" S. aureus

"[...] irresponsabilidade, falta de compromisso e falta se conscientizar [...]" Candida albicans

"[...] esquecimento, falta de atenção, excesso de trabalho [...]" Staphylococcus epidermidis

Observar o não comprometimento da equipe à adequação das orientações influencia na mudança de rotina e emergem questionamentos a respeito das práxis do cuidado em saúde em um ambiente complexo e diferenciado. A percebida resistência em adotar práticas simples para a redução de infecção ainda é fortemente identificada:

\section{"[... ] existe falta de interesse do funcionário [...]" Enterococcus}

falta de adesão dos profissionais de saúde a essa prática acarreta necessidade de reformulação cultural, a fim de valorizar a segurança e a qualidade da assistência (MOTA et al., 2014). Assim, cabe ao enfermeiro gestor identificar as razões desta resistência entre os profissionais em relação às recomendações e proporcionar estratégias educacionais, estimulando e conscientizando os profissionais, incluindo ele, na tentativa de mudar a cultura prevalente. Os microrganismos estão a cada dia mais agressivos e encontrando formas de sobreviver em meio à resistência estimulada, muitas vezes, pelos próprios profissionais de saúde (OLIVEIRA et al., 2012).

O enfrentamento da situação é urgente, falas devem ser substituídas por ações seguras no cuidado a pacientes criticamente enfermos. Ressaltam-se que medidas individuais não são capazes de modificar e manter o comportamento de higienização das mãos pelos profissionais de saúde por tempo prolongado, sendo necessárias ações coletivas e abrangentes (BELELA-ANACLETO et al., 2017). Falhas no processo de higienização das mãos acentuam a condição de risco ao paciente, pois segundo a legislação vigente, o usuário com demanda de cuidados intensivos deverá ser assistido em unidade com infraestrutura adequada e especializada para este fim, proporcionando atuação segura e eficaz da equipe.

\section{CONCLUSÃO}

As mãos são consideradas ferramentas indispensáveis à vida, de modo que na área da saúde desenvolvem papel crucial na promoção e manutenção desta, porém os resultados deste estudo remetem à reflexão sobre o compromisso, a responsabilidade e a ética de profissionais no cuidado ao paciente, 
conferindo segurança a ele e a si. A deficiência na descrição da correta técnica de higienização das mãos, o elevado índice de contaminação nas mãos de profissionais de saúde atuantes em UTI e a afirmação de que o ambiente de trabalho disponibiliza condições para a realização efetiva desta higienização, remete a um perfil profissional de resistência para a não adesão à higienização das mãos, como uma prática simples e primordial para a redução das taxas de infecção hospitalar.

\section{REFERÊNCIAS}

1. ALMEIDA ECB, COSTA ANB, ROSA PB et al. Ações de educação em higienização das mãos como estratégia à segurança do paciente: relato de experiência. REBES, 2017; 7(2): 68-71.

2. BELELA-ANACLETO ASC, SOUSA BEC, YOSHIKAWA JM et al. Higienização das mãos e a segurança do paciente: perspectiva de docentes e universitários. Texto e Contexto Enfermagem, 2013; 22(4): 901-908.

3. BELELA-ANACLETO ASC, PETERLINI MAS, PEDREIRA MLG. Higienização das mãos como prática do cuidar: reflexão acerca da responsabilidade profissional. Revista Brasileira de Enfermagem, 2017; 70(2): 461-464. BRASIL. Agência Nacional de Vigilância Sanitária. Manual Segurança do paciente: Higienização das Mãos. Brasília: Anvisa, 2009.

4. BRASIL. Agência Nacional de Vigilância Sanitária. Higienização das mãos em serviços de saúde. Brasília: Anvisa, 2007.

5. BRASIL. Ministério da Saúde. Portaria No 2.616, de 12 de maio de 1998. Expede na forma de anexos diretrizes e normas para a prevenção e controle das infecções hospitalares. Diário Oficial da União, 1998.

6. CATANEO C, CANINI SRMS, CASTRO PTO et al. Evaluation of the sensitivity and specificity of criteria for isolation of patients admitted to a specialized cancer hospital. Revista Latino-Americana de Enfermagem, 2011; 19(5): $1072-1079$.

7. CORDEIRO VB, LIMA CB. Higienização das mãos como ferramenta de prevenção e controle de infecção hospitalar. Temas em Saúde, 2016; 16(2): 425-444.

8. FIGUEIREDO DA, VIANNA RPT, NASCIMENTO JÁ. Epidemiologia da Infecção Hospitalar em uma Unidade de Terapia Intensiva de um Hospital Público Municipal de João Pessoa-PB. Revista Brasileira de Ciências da Saúde, 2013; 17(3): 233-240.

9. GIAROLA LB et al. Infecção hospitalar na perspectiva dos profissionais de enfermagem: um estudo bibliográfico. Cogitare Enfermagem, 2012; 17(1): 151-157.

10. MINAYO MCS et al. O desafio do conhecimento. São Paulo: Hucitec, 2007.

11. MOTA EC et al. Higienização das mãos: uma avaliação da adesão e da prática dos profissionais de saúde no controle das infecções hospitalares. Revista de Epidemiologia e Controle de Infecção, 2014; 4(1): 12-17.

12. OLIVEIRAAC, PAULAAO. Fatores relacionados à baixa adesão à higienização das mãos na área da saúde: uma reflexão. Ciência, Cuidado e Saúde, 2014; 13(1): 185-190.

13. OLIVEIRA AC, PINTO SA. Participação do paciente na higienização das mãos entre profissionais de saúde. Revista Brasileira de Enfermagem, 2018; 71(2): 259-264.

14. OLIVEIRAAC, SILVA MDM, GARBACCIO JL. Vestuário de profissionais de saúde como potenciais reservatórios de microrganismos: uma revisão integrativa. Texto e Contexto Enfermagem, 2012; 21(3): 684-691.

15. OLIVEIRA HM, SILVA CPR, LACERDA RA. Policies for control and prevention of infections related to healthcare assistance in Brazil: a conceptual analysis. Revista Escola de Enfermagem da USP, 2016; 50(3): 502-508.

16. ROCHA JPJ, LAGES CAS. O Enfermeiro e a prevenção das infecções do sítio cirúrgico. Cadernos UniFOA, 2016; $30: 117-128$.

17. STUBE M et al. O enfermeiro na prevenção de infecções em terapia intensiva. Revista de Enfermagem UFPE on line, 2013; 7 (esp): 6989-6997.

18. VOLQUIND D, BAGATINI A, MONTEIRO GMC et al. Riscos e doenças ocupacionais relacionados ao exercício da anestesiologia. Revista Brasileira de Anestesiologia, 2013; 63(2): 227-232. 